\title{
Recovery of neutrophil apoptosis by ectoine: a new strategy against lung inflammation
}

\author{
Ulrich Sydlik*, Henrike Peuschel*, Adnana Paunel-Görgülü\# ${ }^{*}$, Stefanie Keymel ${ }^{\uparrow}$, \\ Ursula Krämer*, Alexander Weissenberg*, Matthias Kroker*, Samira Seghrouchni\#, \\ Christian Heiss", Joachim Windolf*, Andreas Bilstein ${ }^{+}$, Malte Kelm ${ }^{*}$, \\ Jean Krutmann ${ }^{*,}$ and Klaus Unfried ${ }^{*, \$}$
}

ABSTRACT: The life span of neutrophilic granulocytes has a determining impact on the intensity and duration of neutrophil driven lung inflammation. Based on the compatible solute ectoine, we aimed to prevent anti-apoptotic reactions in neutrophils triggered by the inflammatory microenvironment in the lung.

Neutrophils from chronic obstructive pulmonary disease patients and control individuals were exposed to inflammatory mediators and xenobiotics in the presence or absence of ectoine. The in vivo relevance of this approach was tested in xenobiotic-induced lung inflammation in rats.

The reduction of apoptosis rates of ex vivo-exposed neutrophils from all study groups was significantly restored in the presence of ectoine. However, natural apoptosis rates not altered by inflammatory stimuli were not changed by ectoine. Mechanistic analyses demonstrated the preventive effect of ectoine on the induction of anti-apoptotic signalling. Neutrophilic lung inflammation induced by single or multiple expositions of animals to environmental particles was reduced after the therapeutic intervention with ectoine. Analyses of neutrophils from bronchoalveolar lavage indicate that the in vivo effect is due to the restoration of neutrophil apoptosis.

Ectoine, a compound of the highly compliant group of compatible solutes, demonstrates a reproducible and robust effect on the resolution of lung inflammation.

KEYWORDS: Carbon black, chronic obstructive pulmonary disease, emphysema, granulocytemacrophage colony-stimulating factor, leukotriene $B_{4}$, phosphatidylinositol 3-kinase

$\mathbf{N}$ eutrophilic inflammation of the lung is an important component of the innate immune response against viral and bacterial pathogens [1]. Neutrophilic granulocytes are recruited from the circulation into the airways by immune complexes containing the chemokine CXCL8 (interleukin (IL)-8) which is secreted from inflammatory or epithelial cells [2]. Pathogens are then affected by the release of reactive oxygen species and pathogen destroying enzymes, such as myeloperoxidase, elastase and matrix metalloproteinases. These reactions, however, also have adverse effects on the lung tissue and neutrophilic inflammation is, therefore, strictly regulated so as not to persist after successful pathogen defence. Accordingly, upon entry of neutrophils into the lung, intracellular signalling cascades are being triggered which lead to neutrophil apoptosis and, ultimately, to their removal by macrophagemediated phagocytosis [3].
Apart from biogenic pathogens, lung inflammation can also be induced by occupational or environmental exposure to xenobiotics or by tobacco smoking [4]. Ongoing exposure may then result in chronic lung inflammation and eventually severe lung diseases, such as chronic obstructive pulmonary disease (COPD). For this reason, some therapeutic approaches in COPD focus on the resolution of neutrophilic lung inflammation [5]. Unfortunately, as the most important group of antiinflammatory drugs, corticosteroids, which in other types of inflammation reliably reduce inflammatory cell numbers, are not effective in the therapy of COPD [6]. Studies on the molecular mechanisms of glucocorticoid resistance of neutrophils suggest that oxidative stress from xenobiotic exposure and/or the inflammatory cells themselves have impact on glucocorticoid receptor activity regulated by histone deacetylases [7].

An alternative approach to reduce the number of neutrophils in chronic lung inflammation therefore
AFFILIATIONS

*IUF - Leibniz Research Institute for Environmental Medicine, and \# Dept of Traumatology and Hand Surgery, Heinrich-Heine-University Duesseldorf, and

'Dept of Cardiology, Pneumology, and Angiology, Heinrich-HeineUniversity Duesseldorf, Duesseldorf, and +bitop AG, Witten, Germany. ${ }^{\S}$ Both authors contributed equally.

\section{CORRESPONDENCE}

K. Unfried

IUF - Leibniz Research Institute for Environmental Medicine

Auf'm Hennekamp 50 40225 Duesseldorf Germany E-mail: klaus.unfried@uniduesseldorf.de

Received:

Aug 022011

Accepted after revision:

July 162012

First published online: Oct 252012 
builds on the concept that neutrophil life span may be reduced if anti-apoptotic reactions triggered by the inflammatory microenvironment are prevented [8]. In a pro-inflammatory situation, inflammatory mediators like granulocyte-macrophage colonystimulating factor (GM-CSF) or leukotriene (LT)B $B_{4}$ counteract natural apoptosis $[9,10]$. The activation of PI3-K (phosphatidylinositol 3-kinase) and Akt (protein kinase B) signalling reduces the proteolytic turnover of $\mathrm{Mcl}-1$, the predominant antiapoptotic protein in neutrophils, and thereby delays naturally scheduled apoptosis leading to an increase of the local inflammatory reaction [11]. Accordingly, pharmaceutical strategies have been developed, which target anti-apoptotic signalling via Akt [12]. This signalling cascade, however, has pleiotropic effects in different cell types and pharmacological intervention at this level may have severe side-effects [12].

Compatible solutes are being used by many cells from bacteria up to higher vertebrates in order to counteract extreme situations like osmotic stress, heat or desiccation [13]. These zwitter-ionic substances are known not to disturb physiological processes in a broad concentration range. By interaction with the hydration layer of macromolecules, the presence of these substances appears to promote thermodynamically stable conformations. Based on the biophysical principle of "preferential exclusion" [14] these substances have stabilising effects on macromolecules and are therefore used in heterologous systems, including biotechnical use, as well as for skin care products and dermatological applications with beneficial effects [15]. We have previously shown that ectoine, a compatible solute from Halomonas elongata, is well tolerated when it is instilled in the lungs of rats [16]. In these studies, ectoine reduced mitogen activated protein kinase (MAPK) activation and IL-8 expression in lung epithelial cells in vitro, as well as in animals that were intra-tracheally treated with carbon nanoparticles (CNP) [17]. The elicitation of these endpoints by environmentally relevant model particles proved to be specifically mediated by PI3-K and Akt signalling events [18], indicating that compatible solutes, and ectoine in particular, prevent molecular stress responses mediated by this mechanism.

In the present study we asked whether the compatible solute ectoine is able to prevent stress-induced signalling pathways responsible for the regulation of neutrophil apoptosis, which is also known to be modulated by Akt-dependent signalling [19]. As ectoine is well tolerated when directly applied to the lung of animals, it could be useful for the treatment of neutrophilic lung inflammation and, in particular, COPD. Therefore, we assessed the influence of ectoine on the prevention of apoptosis in neutrophils isolated from healthy volunteers, COPD patients and clinical controls. In a further set of experiments the effects of ectoine on lung neutrophil apoptosis and the persistence of lung inflammation were studied in vivo in animals treated with environmental model particles.

\section{METHODS}

\section{Volunteers and patients}

The group of young healthy volunteers consisted of six males and four females (age 29.9 $\pm 4.2 \mathrm{yrs}$ ). Male patients with stable COPD were compared to male control individuals. Demographic information as well as health status (including COPD criteria) of patients and controls are given in table 1. Both groups were recruited from a cohort of patients with symptoms of coronary artery disease. All subjects were screened by clinical history, physical examination, electrocardiogram at rest and routine chemical analyses. Medication was discontinued on the day of the investigations. Patients with severe chronic heart failure, renal insufficiency (glomerular filtration rate $<30 \mathrm{~mL} \cdot \mathrm{min}^{-1}$ ), a malignant disease, an inflammatory disease as indicated by an increased C-reactive protein $>5 \mathrm{mg} \cdot \mathrm{L}^{-1}$, vasculitis or Raynaud's syndrome were excluded. The study was approved by the local ethics committee on human research of the Heinrich-HeineUniversity Duesseldorf (Duesseldorf, Germany) and written informed consent was obtained from all study subjects before enrolment.

\section{Isolation and treatment of human neutrophils}

Neutrophil isolation and apoptosis measurement were performed as described previously [19]. $2 \mathrm{~h}$ prior to treatment with particles or inflammatory mediators, ectoine (in PBS) or PBS (sham control) were added to the cultures. Cells were then treated with $33 \mu \mathrm{g} \cdot \mathrm{mL}^{-1} \mathrm{CNP}$ in PBS, $300 \mathrm{nM} \mathrm{LTB}_{4}$ (Calbiochem/ Merk, Nottingham, UK) in $0.1 \% \mathrm{EtOH}$, or $20 \mathrm{ng} \cdot \mathrm{mL}^{-1} \mathrm{GM}-\mathrm{CSF}$

\begin{tabular}{|c|c|c|c|}
\hline \multirow[t]{2}{*}{ TABLE 1} & \multicolumn{3}{|c|}{$\begin{array}{l}\text { etails and health status of } \\
\text { ronic obstructive pulmonary } \\
\text { and control subjects }\end{array}$} \\
\hline & COPD & $\begin{array}{l}\text { Control } \\
\text { subjects }\end{array}$ & p-value \\
\hline \multicolumn{4}{|l|}{ Demographics } \\
\hline Subjects $n$ & 10 & 10 & \\
\hline Age yrs & $66.8 \pm 7.5$ & $64.7 \pm 12.4$ & \\
\hline Males & 100 & 100 & \\
\hline Height $\mathrm{m}$ & $1.76 \pm 0.05$ & $1.77 \pm 0.08$ & NS \\
\hline Weight kg & $85.4 \pm 12.4$ & $90.1 \pm 21.2$ & NS \\
\hline Body mass index $\mathrm{kg} \cdot \mathrm{m}^{-2}$ & $27.6 \pm 3.2$ & $28.3 \pm 4.6$ & NS \\
\hline Current smoker & 10 & 0 & \\
\hline Former smoker & 90 & 90 & \\
\hline Pack-yrs & $46.5 \pm 18.3$ & $23.9 \pm 12.2$ & 0.006 \\
\hline \multicolumn{4}{|l|}{ GOLD classification } \\
\hline I & 20 & 0 & \\
\hline$\|$ & 50 & 0 & \\
\hline III & 30 & 0 & \\
\hline IV & 0 & 0 & \\
\hline \multicolumn{4}{|l|}{ Lung function } \\
\hline FVC \% pred & $77 \pm 18$ & $86 \pm 9$ & NS \\
\hline FEV $1 \%$ pred & $62 \pm 20$ & $86 \pm 8$ & 0.008 \\
\hline FEV $1 / F V C \%$ pred & $60 \pm 9$ & $79 \pm 9$ & 0.001 \\
\hline Total lung capacity \% pred & $100 \pm 13$ & $90 \pm 11$ & NS \\
\hline Residual volume \% pred & $151 \pm 29$ & $106 \pm 17$ & 0.003 \\
\hline \multicolumn{4}{|l|}{ Blood parameters } \\
\hline $\mathrm{C}$-reactive protein $\mathrm{mg} \cdot \mathrm{dL}^{-1}$ & $0.46 \pm 0.31$ & $0.50 \pm 0.28$ & NS \\
\hline White blood cell count cells $\cdot \mathrm{L}^{-1}$ & $8.14 \pm 3.34$ & $7.22 \pm 2.70$ & NS \\
\hline \multicolumn{4}{|l|}{ Coronary artery disease } \\
\hline 1 vessel & 30 & 10 & \\
\hline 2 vessels & 0 & 10 & \\
\hline 3 vessels & 70 & 80 & \\
\hline $\begin{array}{l}\text { Data are presented as mean } \pm \mathrm{SD} \\
\text { Initiative for Chronic Obstructive L } \\
\text { pred: \% predicted; FEV } 1 \text { : forced } \mathrm{e}\end{array}$ & $\begin{array}{l}\text { \%, unless oth } \\
\text { g Disease; F } \\
\text { ratory volume }\end{array}$ & $\begin{array}{l}\text { se stated. GC } \\
\text { forced vital } \\
1 \text { s; NS: not s }\end{array}$ & $\begin{array}{l}\text { : Global } \\
\text { oacity; \% } \\
\text { nificant. }\end{array}$ \\
\hline
\end{tabular}


(Cell Signaling Technology, Beverley, MA, USA) in PBS. Cells were harvested after $6 \mathrm{~h}$ (for the analyses of signalling proteins) and $16 \mathrm{~h}$ (for measurement of apoptosis), from the same samples.

\section{Quantification of apoptotic cells}

Blood neutrophils $\left(4 \times 10^{5}\right)$ were suspended in $300 \mu \mathrm{L}$ hypotonic solution containing propidium iodide. The red fluorescence of propidium iodide was measured flow cytometrically (FACScan cytometer; BD Biosciences, San Jose, CA, USA). A minimum of $10^{4}$ events were counted per sample. Western blots and the respective protein preparations were performed as described previously [17, 19].

\section{Particle suspensions and compound solutions}

14-nm diameter CNP (Carbon Black, Printex 90; Degussa, Frankfurt, Germany) and ectoine solution ((S)-2-methyl-1,4,5,6tetrahydropyrimidine-4-carboxylic acid, lipopolysacchride-free, ultrapure 99\%; bitop AG, Witten, Germany) were characterised and prepared as described previously [17].

\section{Animal experiments}

Female Fisher 344 rats (8-weeks old; Charles River Laboratories, Sulzfeld, Germany) were instilled intra-tracheally with $0.4-\mathrm{mL}$ particle suspension, ectoine solution (doses indicated in the figures) or PBS under inhalation anaesthesia (isoflurane 5\%, $2 \mathrm{~min}$ ). Animals were sacrificed by exsanguination under pentobarbital anaesthesia, after the indicated periods. Lung lavages were performed using $4 \times 5 \mathrm{~mL}$ PBS. Differential cell counts were performed after Giemsa/May-Grünwald staining of lavage cells. Cell free lavage fluids were used for cytokine assays. Lung tissues were minced, shock frozen and stored at $-80^{\circ} \mathrm{C}$ until further use. All animal experiments were performed after relevant permission according to German animal protection laws. Myeloperoxidase was measured by ELISA using a rat myeloperoxidase kit (Cell Sciences, Canton, MA, USA). For apoptosis measurements, lavage cells from each animal were suspended in $1 \mathrm{~mL}$ PBS and subjected to Percoll centrifugation (Biochrom, Berlin, Germany). Cell pellets were washed once with PBS and resuspended in $300 \mu \mathrm{L}$ hypotonic solution ( $0.1 \%$ sodium citrate, $0.1 \%$ Triton $X 100$ ) containing $50 \mu \mathrm{g} \cdot \mathrm{mL}^{-1}$ propidium iodide and subsequently subjected to fluorescence measurements.

\section{Statistics}

Due to the log normal distribution of data, statistical analysis was performed after logarithmic transformation. ANOVA was used throughout (proc mixed, SAS 9.2; SAS Institute Inc., Cary, NC, USA). If appropriate, repeated measures of ANOVA were used (proc glm, SAS 9.2; SAS Institute Inc.). Main effects and interactions were tested. Changes were estimated relative to the respective controls and are given with their 95\% confidence interval $(95 \% \mathrm{CI})$. A result was considered as statistically significant when the $\mathrm{p}$-value was $<0.05$. Multiple testing was avoided. The main hypothesis per experiment was tested. In the rare cases of two tested hypotheses per experiment, Bonferroni correction was applied $(p=0.025)$. Each experiment is graphically represented by one figure. Data are depicted as mean \pm SD.

\section{RESULTS}

Ectoine restores spontaneous apoptosis in isolated human neutrophils

Peripheral blood neutrophils were isolated from young healthy volunteers and subsequently subjected to treatment that mimics the inflammatory situation in the lung (fig. 1a and b). Besides the inflammatory mediators GM-CSF $\left(20 \mathrm{ng} \cdot \mathrm{mL}^{-1}\right)$ and $\mathrm{LTB}_{4}(300 \mathrm{nM}), \mathrm{CNP}\left(33 \mu \mathrm{g} \cdot \mathrm{mL}^{-1}\right)$, which is accepted as representative for ultrafine particulate air pollution [20], was used for cell treatment. All these pro-inflammatory stimulants significantly reduced the percentage of apoptotic neutrophils by a factor of 0.61 (95\% CI $0.53-0.69) \quad 16 \mathrm{~h}$ after exposure (fig. 1a and b). This effect was equal for the three stimulants used. The pre-treatment of neutrophils with $1 \mathrm{mM}$ of ectoine $2 \mathrm{~h}$ prior to inflammatory mediators or particles nearly completely restored apoptosis rates $(0.82,95 \%$ CI $0.82-0.99)$, independent from the anti-apoptotic agent and were highly significant (repeated measures ANOVA, F-test $\mathrm{p}<0.0001$ ).

In general, neutrophils do not respond well to anti-inflammatory therapies like corticosteroid treatment [6] and, in the case of COPD, may even become resistant to this kind of therapy [7]. We therefore repeated the experiments with blood neutrophils from patients with COPD as well as from clinical control individuals (fig. $1 \mathrm{c}$ and d). Although higher background apoptosis rates were observed in these groups, all three inflammatory stimulants reduced the number of apoptotic cells from clinical controls and patients with COPD (0.49, $95 \%$ CI $0.42-0.59$ and $0.58,95 \%$ CI $0.50-0.67$, respectively). Again, this reduction was much smaller after ectoine pretreatment in cells from both groups $(0.87,95 \%$ CI $0.80-0.95$ for patients with COPD and $0.84,95 \%$ CI $0.79-0.90$ for controls). The effects proved to be significant $(p<0.0001)$ and independent of the inflammatory stimulant and from COPD status (repeated measures ANOVA, F-test).

\section{Ectoine acts as a preventive at the level of membrane- coupled signalling}

The cellular and molecular mechanisms underlying the effect of ectoine on apoptosis were further investigated in neutrophils from healthy volunteers treated with carbon particles $\left(33 \mu \mathrm{g} \cdot \mathrm{mL}^{-1}\right)$. Increasing concentrations of ectoine gradually restored apoptosis rates (fig. 2a). The ectoine effect was significant (repeated measures ANOVA, F-test, $\mathrm{p}<0.001$ ). To investigate whether ectoine itself has any pro-apoptotic activity, human neutrophils from three male and two female human donors were exposed to a broad dose range of ectoine (0.01-10 mM) (fig. 2b). Compared to sham treated controls, no ectoine effect was detected $(p=0.3024)$.

As a next step, the effects of ectoine on anti-apoptotic signalling were investigated. For this purpose, neutrophils isolated from healthy volunteers were analysed for Akt phosphorylation at $\operatorname{Ser}^{473}$ as a specific downstream event of PI3-K signalling. Phosphorylation levels in comparison to total Akt amounts in Western blot analyses showed a significant (repeated measures ANOVA, F-test, $\mathrm{p}<0.0001$ after Bonferroni correction) activation of this pathway by CNP, GM-CSF and $\mathrm{LTB}_{4}$ (fig. 2c), indicating that all anti-apoptotic stimuli trigger the same anti-apoptotic pathway. Importantly, in all three cases, pre-incubation of human neutrophils with ectoine prevented the activation of this pathway. In these cells, phosphorylation levels were not significantly different from those before application of the anti-apoptotic stimuli. The ectoine effect was significant (repeated measures ANOVA, Ftest, $\mathrm{p}=0.012$ after Bonferroni correction). As a result of Akt activation, amounts of anti-apoptotic Mcl-1 were increased 


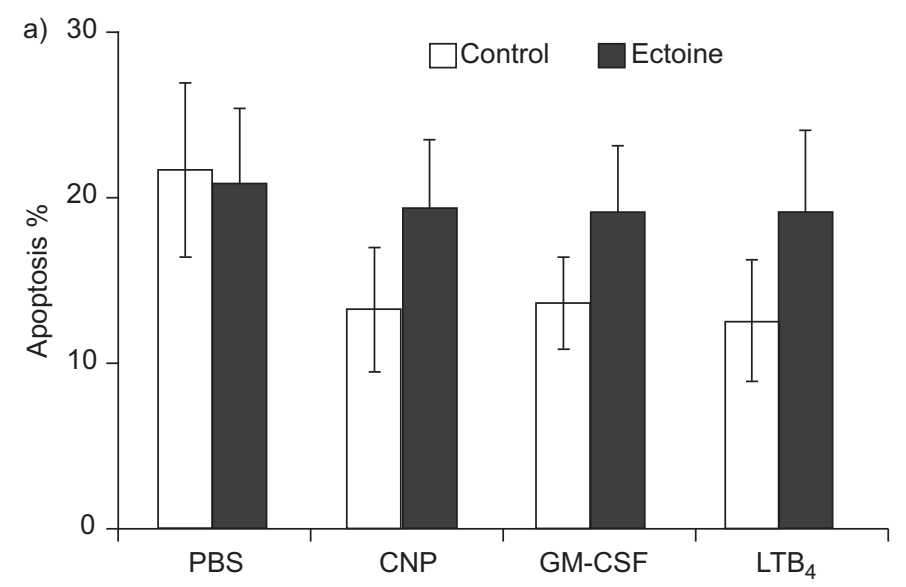

b)

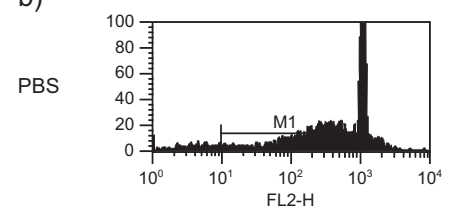

CNP

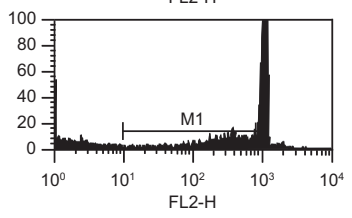

GM-CSF
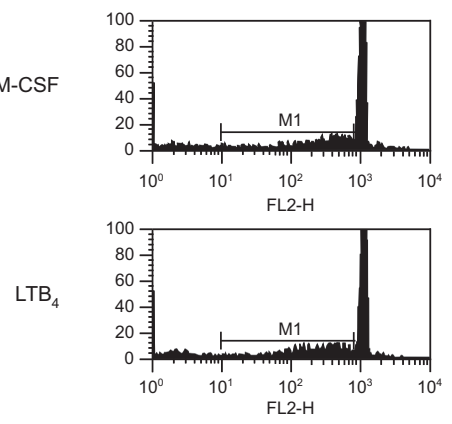

Ectoine
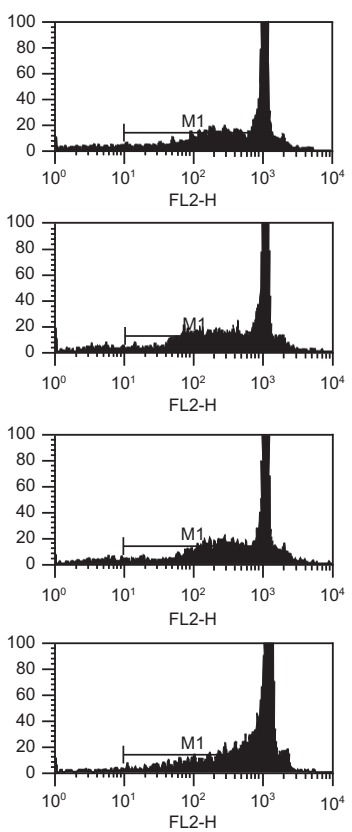
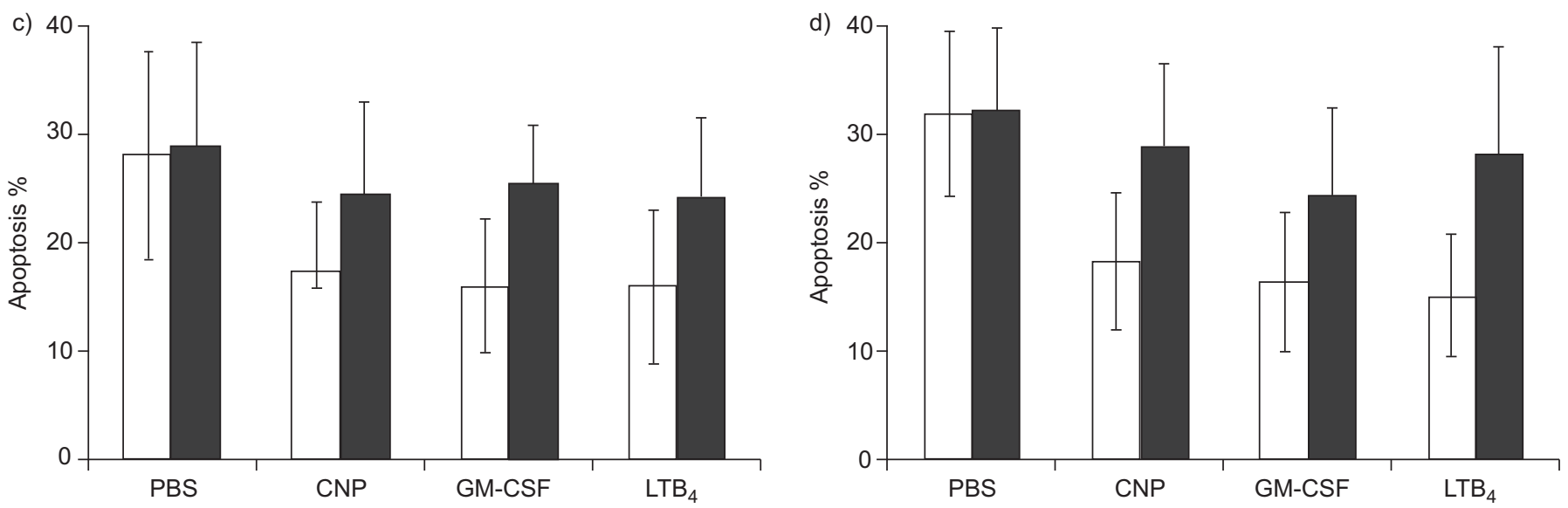

FIGURE 1. Ectoine restores apoptosis rates in isolated human neutrophils. a) Apoptotic cells from neutrophils isolated from 10 young healthy volunteers and b) representative histogram plots of sub-G1 measurements. Cells were pre-treated with $1 \mathrm{mM}$ ectoine for $2 \mathrm{~h}$ followed by $16 \mathrm{~h}$ of treatment with $33 \mu \mathrm{g} \cdot \mathrm{mL}^{-1}$ carbon nanoparticles (CNP), $300 \mathrm{nM}$ leukotriene (LT)B $4,20 \mathrm{ng} \cdot \mathrm{mL}^{-1}$ granulocyte-macrophage colony-stimulating factor (GM-CSF) or PBS. Apoptotic cells from neutrophils isolated from patients with c) chronic obstructive pulmonary disease or d) control (each $n=10$ ) treated as described in (a).

after treatment with CNP, GM-CSF and $\mathrm{LTB}_{4}$ (3.0 times, 95\% CI 2.0-4.4). Pre-treatment with ectoine largely prevented this key anti-apoptotic event (1.6 times, 95\% CI 1.0-2.4) (fig. 2d). Again, the ectoine effect was significant (repeated measures ANOVA, F-test, $\mathrm{p}=0.0173$ ).

\section{Ectoine restores apoptosis rates in vivo and reduces pre- existing neutrophilic inflammation}

Next we investigated whether ectoine also has preventive effects in vivo on neutrophilic inflammation that was induced in lungs of Fischer 344 rats by intra-tracheal instillation of xenobiotic model particles. As depicted in figure 3a, inflammation was first induced by a single instillation of $2.5 \mathrm{mg} \cdot \mathrm{kg}^{-1}$ CNP followed by two consecutive ectoine applications given at day 1 and day 2. At day 3, bronchoalveolar lavage (BAL) was performed and specimens were analysed for neutrophil apoptosis, as well as inflammatory parameters. As figure $3 \mathrm{~b}$ shows, CNP exposure alone significantly reduced the percentage of apoptotic cells $(0.59,95 \%$ CI $0.45-0.78)$ indicating that anti-apoptotic mechanisms had been elicited by CNP and/or CNP-triggered inflammatory mediators. In CNP-exposed animals that were subsequently treated twice with $0.1 \mathrm{mM}$ or $1 \mathrm{mM}$ ectoine, an improvement of neutrophil apoptosis was observed $(0.64,95 \%$ CI $0.48-0.84$ or $0.82,95 \%$ CI $0.62-1.07$, respectively). The ectoine effect proved to be statistically significant $(p=0.0215$, ANOVA, F-test).

Importantly, advanced neutrophil apoptosis correlated with the significant reduction of lung inflammation measured as a percentage of neutrophils and the amount of cinc-1 (neutrophil recruiting IL-8 homologue) in BAL (fig. $3 c$ and d). After CNP exposure, 4.4 times (95\% CI 3.2-5.6) as many neutrophils and 

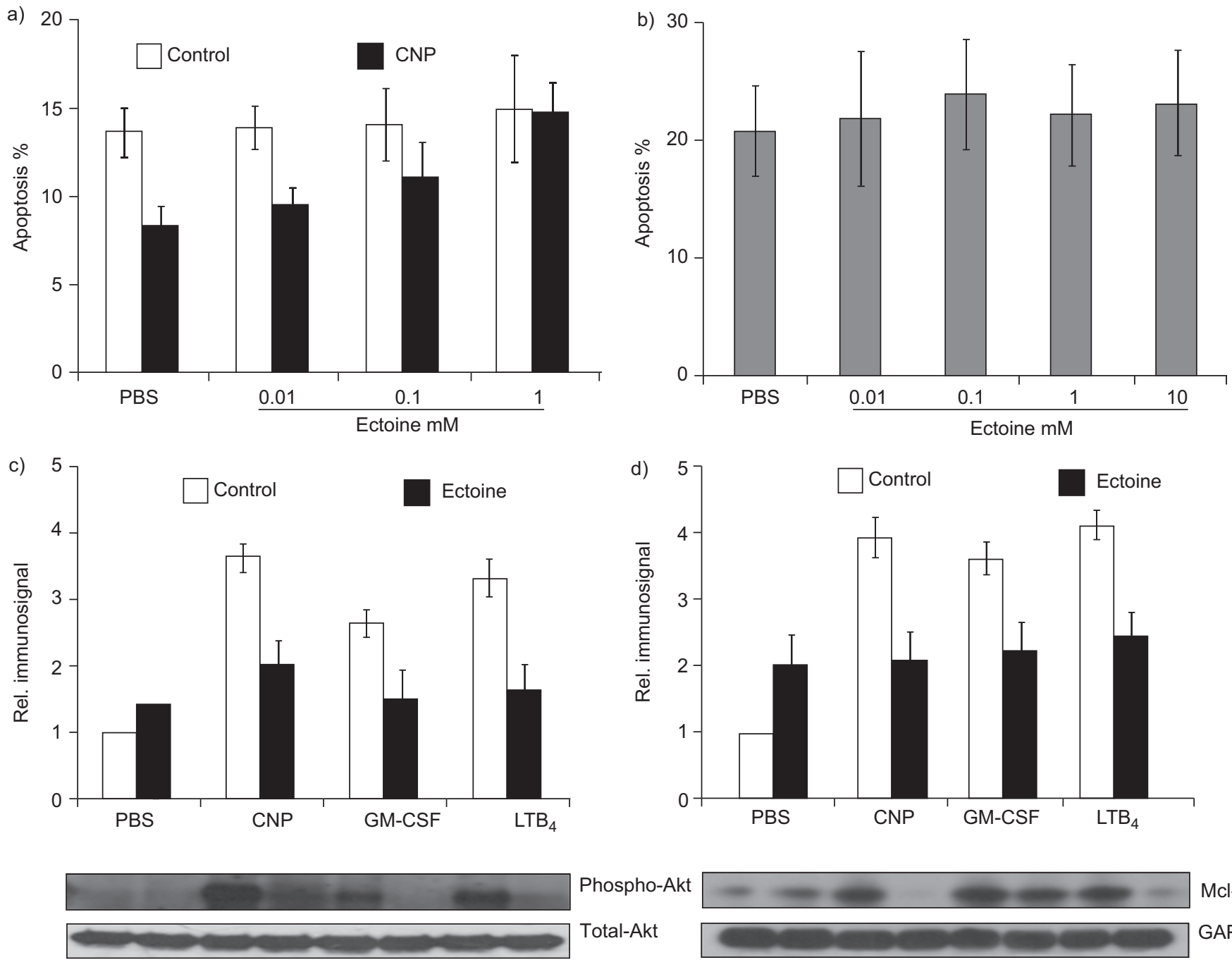

Phospho-Akt

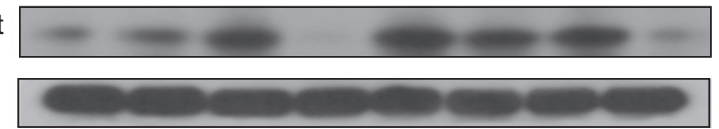

Mcl-1

Total-Akt

GAPDH

FIGURE 2. Ectoine prevents anti-apoptotic reactions via Akt-mediated signalling. a) Apoptotic cells from neutrophils isolated from five young healthy volunteers. Cells were initially treated for $2 \mathrm{~h}$ with the indicated doses of ectoine followed by $16 \mathrm{~h}$ of treatment with $33 \mu \mathrm{g} \cdot \mathrm{mL}^{-1}$ carbon nanoparticles (CNP) or PBS. b) Apoptotic cells from neutrophils isolated from five volunteers treated for $18 \mathrm{~h}$ with the indicated doses of ectoine alone. Quantification and representative immunoblots of $\mathrm{c}$ ) protein kinase B (Akt) and d) myeloid leukaemia cell differentiation protein (Mcl-1) in neutrophils $6 \mathrm{~h}$ after treatments with PBS, $33 \mu \mathrm{g} \cdot \mathrm{mL}^{-1} \mathrm{CNP}, 20 \mathrm{ng} \cdot \mathrm{mL}^{-1} \mathrm{granulocyte}-\mathrm{macrophage}$ colonystimulating factor (GM-CSF) or $300 \mathrm{nM}$ leukotriene (LT)B 4 . Ectoine pre-treatment was $1 \mathrm{mM}$ for $2 \mathrm{~h}$. GAPDH: glyceraldehyde-3 phosphate dehydrogenase.

3.1 times (95\% CI 2.4-4.0) higher concentrations of cinc-1 could be observed in BAL compared to no CNP exposure. After additional application of $1 \mathrm{mM}$ ectoine, the elevation was reduced to 3.1 times (95\% CI 2.2-4.4) for the number of neutrophils and 2.1 times (95\% CI 1.6-2.6) for the concentration of cinc-1. The ectoine effect was significant for both outcomes (ANOVA, F-test, $\mathrm{p}=0.0005$ ). This therapeutic effect was confirmed at the level of neutrophil activation by measuring myeloperoxidase (fig. 3e). Myeloperoxidase levels were increased 19.6 times $(95 \%$ CI $11.6-41.8)$. This increase was reduced to 10.3 times (95\% CI 5.3-60.8) after additional treatment with ectoine (ANOVA, F-test, $\mathrm{p}=0.0633$ ). We had previously reported that CNP activates the MAPK extracellular signalregulated protein kinases 1 and 2 (Erk1/2) in lung epithelial cells [21]. Assessment of Erk1/2 phosphorylation in lung homogenates of animals revealed that ectoine also significantly diminished this tissue response $(\mathrm{p}=0.0166$, ANOVA, F-test) (fig. 3e). In aggregate, these observations strongly indicate that ectoine can reduce a pre-existing neutrophilic lung inflammation.

\section{Ectoine effects during repetitive xenobiotic exposure in vivo}

In a real life scenario, however, chronic inflammation would result from continuous exposure rather than from single exposure to xenobiotics. In order to mimic this situation, we administered ectoine $(1 \mathrm{mM})$ twice after one, two or three instillations of CNP (fig. 4a). In these experiments, neutrophil apoptosis was significantly reduced by each CNP application to $66.0 \%$ (95\% CI $53.9-$ $80.9 \%)$, and was restored each time (85.8, 95\% CI 69.6-105.9\%) when this treatment was followed by two instillations of $1 \mathrm{mM}$ ectoine. The restoration was equally pronounced in all three exposure scenarios, no significant interaction between exposure 
a)
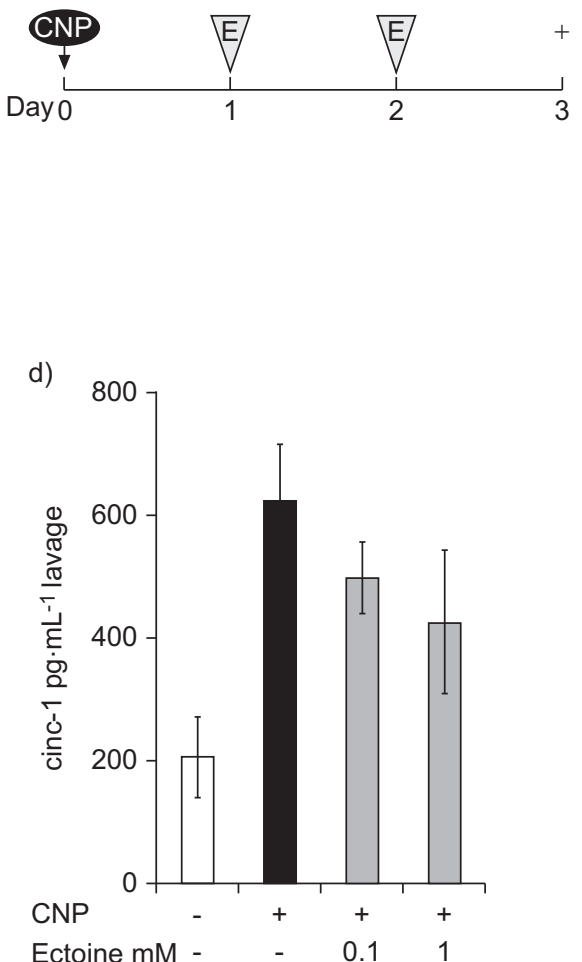

b)

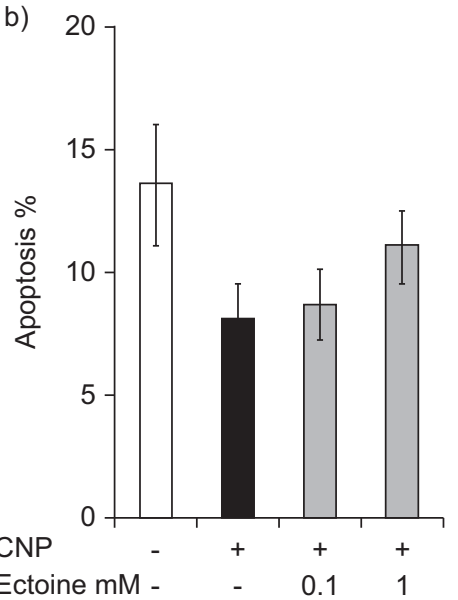

e)

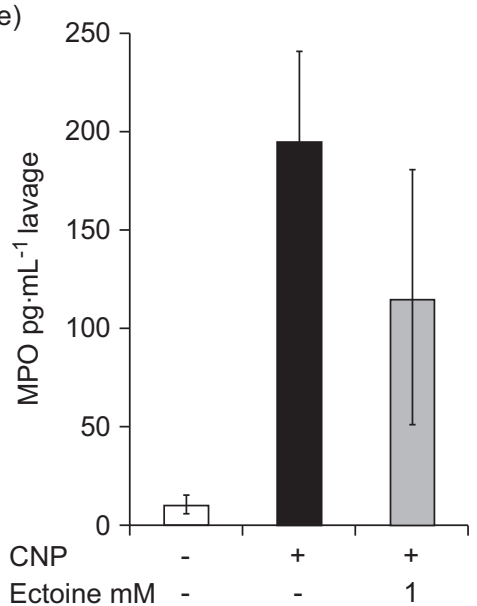

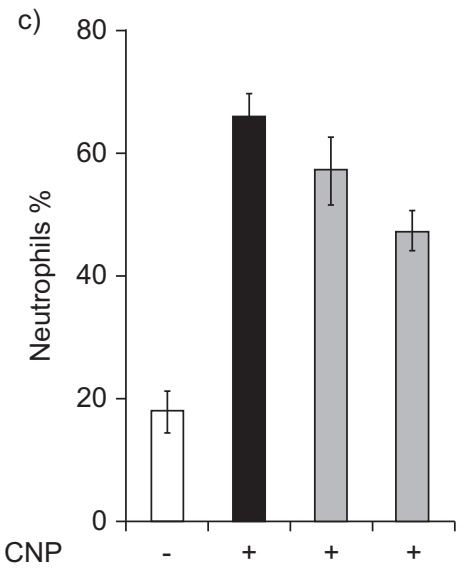

Ectoine $\mathrm{mM}-\quad-\quad 0.1 \quad 1$

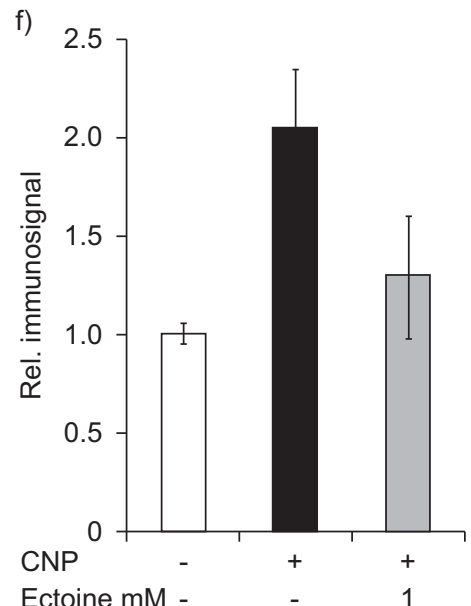

FIGURE 3. Ectoine reduces inflammation by shortening neutrophilic life span in lung inflammation in vivo. a) Setup of animal experiment with Fischer 344 rats. Carbon nanoparticles (CNP; $2.5 \mathrm{mg} \cdot \mathrm{kg}^{-1}$ bodyweight) and ectoine (E) were intra-tracheally instilled. PBS served as sham for both treatments. b) Apoptosis of neutrophils isolated from bronchoalveolar lavage (BAL) of four animals treated according to (a). BAL parameters c) neutrophils and d) cinc-1 of seven animals treated according to (a). e) Myeloperoxidase (MPO) in BAL and f) phosphorylation of extracellular signal-regulated protein kinases 1 and 2 (Erk1/2) in lung tissue of five animals exposed according to the scenario d2 in figure 4.

scenario and ectoine effect was detected. The ectoine effect was significant (ANOVA, F-test, $\mathrm{p}=0.0091$ ) (fig. 4b).

The preventive effect of ectoine therapy was also observed at the level of inflammation. Under all experimental conditions, ectoine significantly reduced neutrophil numbers and cinc-1 levels in BAL of CNP-treated rats (fig. 4c and d). These effects were significant $(p<0.0001$, ANOVA, F-test $)$ for both endpoints and the dimension of the effect was equally pronounced in all three exposure scenarios. The therapeutic effect of ectoine was also demonstrated by comparative measurement of inflammatory cytokines in BAL from animals with pre-existing inflammation using membrane-coupled cytokine arrays. Figure $4 \mathrm{e}$ demonstrates that, in addition to cinc-1, several other pro-inflammatory factors (tumour necrosis factor- $\alpha$, GMCSF, IL- $1 \alpha$, IL-1 $\beta$, IL-4, IL- 6 and cinc-2), which were elevated due to the xenobiotic particle treatment, were significantly reduced upon ectoine treatment to $41.2 \%$ (95\% CI 31.4-54.1\%, repeated measures ANOVA, F-test $\mathrm{p}<0.0001)$ of the value without ectoine, while interferon- $\gamma$ and anti-inflammatory
IL-10 only changed to $81 \%(95 \%$ CI $48.0-137 \%)$ of the value before treatment, which was not significant.

\section{Persistent reduction of neutrophil numbers in rat lungs by ectoine}

Next, we studied whether the preventive effect of ectoine persists during the course of inflammation. For that purpose, $2.5 \mathrm{mg} \cdot \mathrm{kg}^{-1}$ of CNP was injected once, either with or without ectoine, in the lungs of animals. Animals were sacrificed at different time-points from $12 \mathrm{~h}$ up to 1 week $(168 \mathrm{~h})$ after exposure and BAL was obtained. Interestingly, the effect of ectoine significantly changed over time (ANOVA, interaction time $\times$ ectoin effect, F-test $\mathrm{p}<0.0001) .12 \mathrm{~h}$ and $24 \mathrm{~h}$ after the application of $\mathrm{CNP}$, ectoine did not significantly reduce the number of lung neutrophils in particle-treated rats (fig. 5a). In marked contrast, from $48 \mathrm{~h}$ up to $168 \mathrm{~h}$ the reduction of neutrophils by ectoine was significant $(p=0.002)$. The number of neutrophils without ectoine treatment was 1.2 times $(95 \% \mathrm{CI}$ 1.1-1.4) higher than that with treatment with no further changes over time. Similarly, from $48 \mathrm{~h}$ onwards, cinc- 1 concentrations 


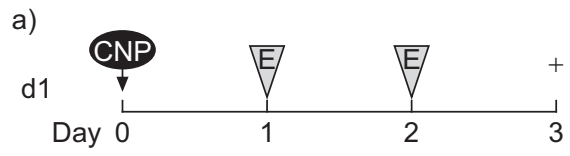

b)

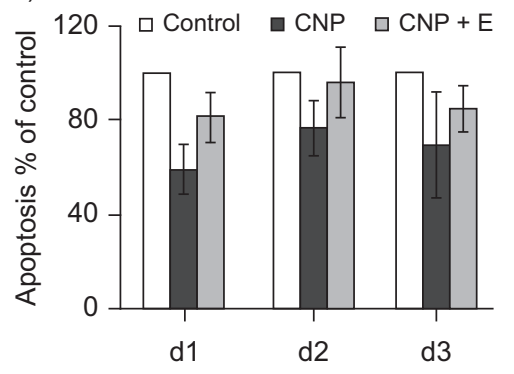

$\mathrm{d} 2$

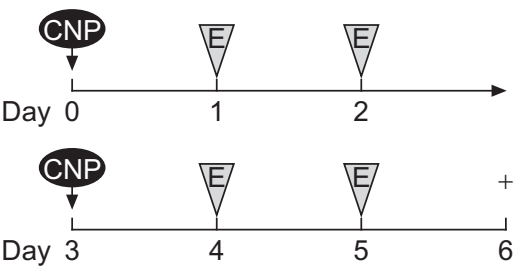

d3

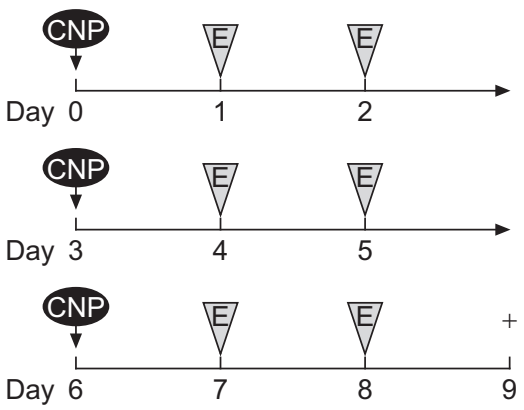

d)

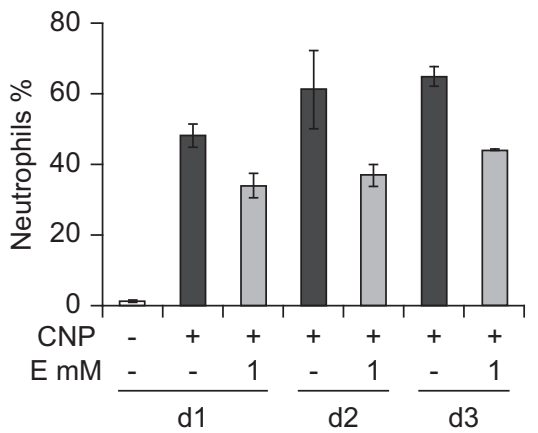

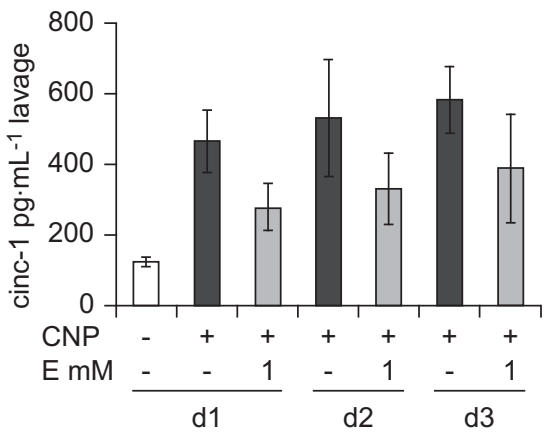

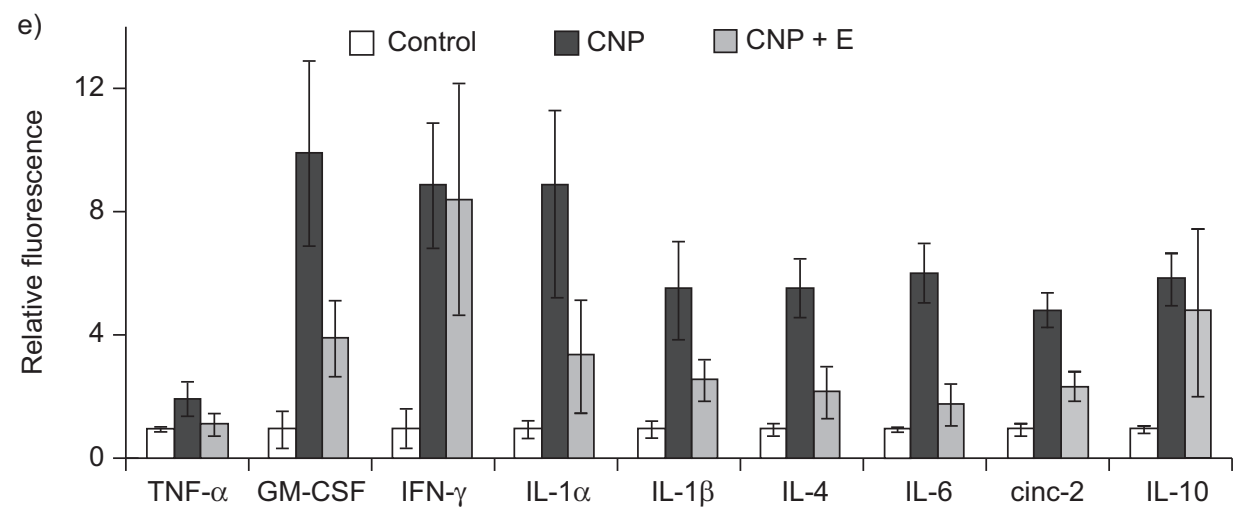

FIGURE 4. Enhanced apoptosis rates induced by repetitive exposure to carbon nanoparticles (CNP) is reduced by ectoine (E). a) Different designs of animal experiments: d1, d2 and d3. Fischer 344 rats were instilled once, twice or three times with CNP (2.5 $\mathrm{mg} \cdot \mathrm{kg}^{-1}$ bodyweight) and subsequently treated twice after each CNP instillation with E, or PBS as sham for both treatments. b) Relative apoptosis rates of neutrophils isolated from animals treated according to d1 ( $n=4)$, d2 (control $n=3$, $\mathrm{CNP}+\mathrm{PBS} \mathrm{n}=5, \mathrm{CNP}+\mathrm{E} \mathrm{n}=5$ ) and $\mathrm{d} 3$ (control $\mathrm{n}=3, \mathrm{CNP}+\mathrm{PBS} \mathrm{n}=6, \mathrm{CNP}+\mathrm{E} \mathrm{n}=3$ ). Bronchoalveolar lavage parameters $\mathrm{c}$ ) neutrophils and $\mathrm{d}$ ) cinc-1 from animals repeatedly treated with CNP and $1 \mathrm{mM} \mathrm{E}$ according to designs $d 1(n=5), d 2(n=5)$, and $d 3(C N P+P B S n=6, C N P+E n=3)$. e) Cytokine pattern of BAL from control animals ( $n=3)$, animals treated according to $d 1$ with $C N P(n=4)$, or treated according to d1 with $C N P+E(n=5)$. TNF- $\alpha$ : tumour necrosis factor- $\alpha$; GM-CSF: granulocyte-macrophage colonystimulating factor; IFN- $\gamma$ : interferon- $\gamma$; IL: interleukin.

in BAL were 2.5 (95\% CI 2.0-3.08) times higher after CNP treatment than after additional ectoine treatment $(\mathrm{p}<0.0001)$ (fig. 5b).

\section{DISCUSSION}

\section{Ectoine treatment prevents anti-apoptotic reactions in human neutrophils}

Chronic exposure to inhalable xenobiotics causes neutrophilic lung inflammation leading to emphysema and COPD [22]. In this case, xenobiotics trigger the release of pro-inflammatory cytokines and chemokines, which lead to an influx and activation of neutrophils [23]. Under these circumstances and regardless of the inducing agent, chronic neutrophilic inflammation is the key pathogenic mechanism in a vicious cycle of necrotic tissue damage and increased recruitment and activation of neutrophils [24]. The neutrophilic inflammation remains or, due to ongoing tissue destruction, is aggravated, even when the inducing agent is removed, e.g. after cessation of smoking [4]. In this inflammatory scenario neutrophils come into contact with inflammatory mediators and also with the xenobiotics present during ongoing exposure. Neutrophil life span may be extended not only by cellular factors but also by the xenobiotics themselves.

In line with this concept, our data clearly demonstrate that spontaneous apoptosis of human neutrophils is counteracted not only by GM-CSF and $\mathrm{LTB}_{4}$ but also by ultrafine carbon 

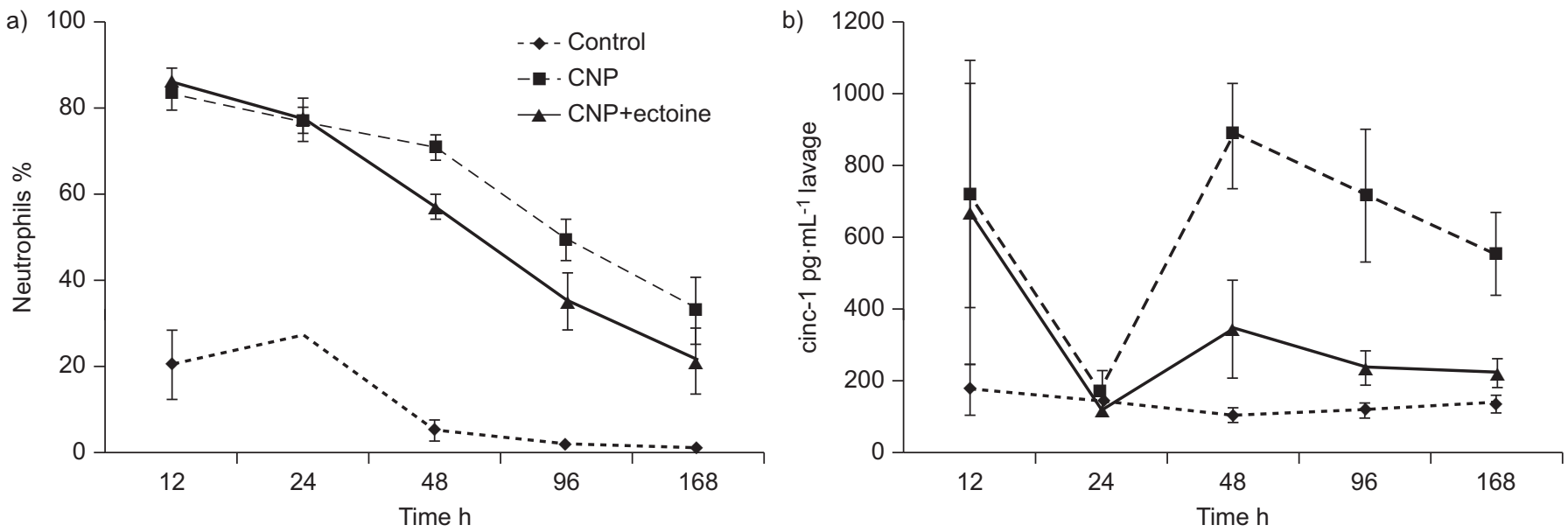

FIGURE 5. Ectoine effects on the resolution of carbon nanoparticle (CNP)-induced lung inflammation. a) Neutrophils and b) cinc-1 from Fischer 344 rats intra-tracheally instilled with $2.5 \mathrm{mg} \cdot \mathrm{kg}^{-1}$ body weight in the presence or absence of $1 \mathrm{mM}$ ectoine. Control animals were treated with $0.4 \mathrm{~mL}$ PBS. Animals ( $\mathrm{n}=3-5$ per group per time-point) were sacrificed and lavaged at the indicated time intervals after instillation.

particles which are a main constituent of particulate air pollution [20]. CNP in several cells are able to induce oxidative stress which triggers membrane signalling pathways [25]. Oxidative stress has been described as a trigger of anti-apoptotic pathways in neutrophils [26]. Our data demonstrate a direct anti-apoptotic effect of environmentally relevant inhalable particles on human neutrophils which may contribute to the persistence of the inflammation during ongoing exposure.

Moreover, the present study provides compelling evidence that the compatible solute ectoine is able to prevent anti-apoptotic reactions of neutrophils induced by inflammatory stimulants. This conclusion is based on the following observations. 1) Antiapoptotic reactions of neutrophils isolated from young healthy volunteers induced by CNP as environmentally relevant model particles or by inflammatory mediators are completely prevented. 2) A striking prevention of anti-apoptotic reactions by ectoine was observed when investigating neutrophils from male COPD patients, as well as from clinical control individuals. 3) The activation of anti-apoptotic membrane-coupled signalling pathways by inflammatory stimulants in neutrophils is prevented in the presence of ectoine.

\section{Ectoine acts as a preventive rather than in a pro-apoptotic}

As ectoine did not alter apoptosis rates of neutrophils which were not stimulated by CNP or inflammatory factors, we conclude that ectoine does not act in a pro-apoptotic. Instead, our data are consistent with the assumption that ectoine has the capacity to prevent anti-apoptotic mechanisms that are known to be activated within the inflammatory microenvironment.

The cell signalling cascade involving PI3-K and Akt has been described as the key mechanism for the anti-apoptotic effects of $\mathrm{LTB}_{4}$ and GM-CSF $[9,10]$. The same pathway was identified to be triggered by CNP-induced oxidative stress [25, 27]. Interestingly, ectoine has been shown to prevent stress-induced membrane-dependent signalling cascades in some systems. In human keratinocytes, the ultraviolet $\mathrm{A}$ radiation stress response can be reduced by ectoine pre-treatment through mechanisms which involve the prevention of second messenger ceramide from cell membrane lipid rafts [28]. In addition, the compatible solutes ectoine and hydroxyectoine have recently been found to act on lipid monolayers and bilayers and to affect fluidity of membranes [29]. The reduction of Akt signals to control, resulting in diminished $\mathrm{Mcl}-1$ levels, indicates that this membrane-coupled pathway is the target of the preventive effect of ectoine in neutrophils as well as in epithelial cells.

\section{Ectoine as a therapeutic strategy to reduce ongoing neutrophilic lung inflammation}

The key question for the therapeutic usefulness of ectoine in lung inflammation is whether the prevention of delayed neutrophil apoptosis has the potential to reduce the neutrophilic infiltrate during the course of ongoing inflammation. In this regard we have clearly shown that in the in vivo model of carbon particle-induced lung inflammation, ectoine restored neutrophil apoptosis rates and at the same time reduced lung inflammation.

In humans, however, the situation might be more complex. Therapeutic or preventive strategies have to cope with the fact that such interventions may be counteracted by self-perpetuating mechanisms of the inflammation itself and/or by continuous exposure from air pollution. In the present study, ectoine was found to reproducibly prevent delayed apoptosis and to reduce inflammation in animals that had been repetitively exposed to CNP. Therefore, we believe that ectoine can reduce neutrophilic lung inflammation under conditions which may be representative for the real life situation in humans.

We also showed that the effect of ectoine persists over the whole period of lung inflammation elicited by a single particle exposure. Interestingly, the general pattern of the release of neutrophil-recruiting cinc- 1 in BAL is consistent with a biphasic response. This kinetic may be best explained by different cell types known to produce this chemokine. During the early recruiting phase, cinc- 1 is likely to be mainly produced by epithelial cells and macrophages, while at later time-points the gradually growing and ultimately dominating number of neutrophils may be responsible for cinc- 1 release. 
This would imply that ectoine affects not only lung epithelial cells, as described previously [17], but neutrophils in particular. Irrespective of the cinc-1 expression pattern, these in vivo data demonstrate a highly reproducible, robust and persistent effect of ectoine on neutrophil numbers and cinc-1 levels, resulting in an accelerated resolution of lung inflammation.

Neutrophil recruitment does not appear to be directly affected by ectoine. At early time-points after the induction of inflammation (12 h and $24 \mathrm{~h}$ ), when neutrophil numbers are dominated by chemokine mediated influx, no ectoine effects can be observed in exposed animals. Later, however, when differences in apoptosis rates are considered to influence neutrophilic lung inflammation, the effect of ectoine becomes obvious. These data corroborate findings of earlier studies in which we observed that rapid neutrophil recruitment within $4 \mathrm{~h}$ of lipopolysaccharide application is not influenced by ectoine [17]. Both results demonstrate that ectoine does not suppress the neutrophil influx that is necessary for the defence of pathogens.

The CNP exposure scenario chosen for the recent experiments was primarily designed to rapidly induce severe inflammation. It employed high doses of CNP which, in the human lung, would most likely result from a cumulative process requiring years of environmental exposure. Therefore, we believe that the exposure regimen used in the present study overestimates rather than underestimates human exposure scenarios. It is therefore not unlikely that patients who are continuously exposed to environmental air pollution may also benefit from a regular application of compatible solutes that have been described as a very compliant group of natural substances with no known side-effects in humans at present [30]. To date, inhalation studies with humans inhaling ectoine have not been published. As a first approach for the treatment of chronic neutrophilic lung inflammation, feasibility as well as efficacy studies have to be performed.

In conclusion, the data presented here demonstrate that instillation of the compatible solute ectoine in the lungs of animals suffering from neutrophilic inflammation induced by environmental model particles can exert significant therapeutic effects. Ectoine appears to act by preventing anti-apoptotic reactions and reducing the life span of lung infiltrating neutrophils. The ectoine effects were observed regardless of whether neutrophil apoptosis was delayed by xenobiotics or by pro-inflammatory factors, and whether isolated human blood neutrophils (from patients, controls or volunteers) or lung neutrophils recruited after exposition to carbon particles were studied. We therefore propose that compatible solutes such as ectoine may be effectively used in clinical settings for the treatment of neutrophilic lung inflammation.

\section{SUPPORT STATEMENT}

This study was supported by project funding from the Ministerium für Umwelt Naturschutz und Reaktorsicherheit (BMU), Deutsche Forschungsgemeinschaft (GRK 1427 and SFB 728), Zukunftswettbewerb NRW, and bitop AG.

\section{STATEMENT OF INTEREST}

Statements of interest for U. Krämer, A. Bilstein, J. Krutmann and $\mathrm{K}$. Unfried, and the study itself can be found at www.erj.ersjournals. $\mathrm{com} / \mathrm{site} / \mathrm{misc} /$ statements.xhtml

\section{ACKNOWLEDGEMENTS}

The excellent technical assistance of R. Wirth and W. Brock, as well as the professional help with animal keeping of S. Martin and P. Gro $\beta$ (all IUF - Leibniz Research Institute of Environmental Medicine, Duesseldorf, Germany) are gratefully acknowledged.

\section{REFERENCES}

1 Chaudhuri N, Sabroe I. Basic science of the innate immune system and the lung. Paediatr Respir Rev 2008; 9: 236-242.

2 Bergion DA, Reeves EP, Meleady P, et al. $\alpha-1$ Antitrypsin regulates human neutrophil chemotaxis induced by soluble immune complexes and IL-8. J Clin Invest 2010; 120: 4236-4250.

3 Serhan CN, Savill J. Resolution of inflammation: the beginning programs the end. Nat Immunol 2005; 6: 1191-1197.

4 MacNee W. Pathogenesis of chronic obstructive pulmonary disease. Proc Am Thorac Soc 2005; 2: 258-266.

5 Beeh KM, Beier J. Handle with care: targeting neutrophils in chronic obstructive pulmonary disease and severe asthma? Clin Exper Allergy 2006; 36: 142-157.

6 Barnes PJ, Adcock IM. Glucocorticoid resistance in inflammatory diseases. Lancet 2009; 73: 1905-1917.

7 Ito K, Yamamura S, Essilfie-Quaye S, et al. Histone deacetylase 2mediated deacetylation of the glucocorticoid receptor enables NFkappaB suppression. J Exp Med 2006; 203: 7-13.

8 Duffin R, Leitch AE, Fox S, et al. Targeting granulocyte apoptosis: mechanisms, models, and therapies. Immunol Rev 2010; 236: 28-40.

9 Klein JB, Rane MJ, Scherzer JA, et al. Granulocyte-macrophage colony-stimulating factor delays neutrophil constitutive apoptosis through phosphoinositide 3-kinase and extracellular signal-regulated kinase pathways. J Immunol 2000; 164: 4286-4291.

10 Petrin D, Turcotte S, Gilbert A-K, et al. The anti-apoptotic effect of leukotriene $\mathrm{B}_{4}$ in neutrophils: a role for phosphatidylinositol 3kinase, extracellular signal-regulated kinase and Mcl-1. Cell Signal 2005; 18: 479-487.

11 Dzhagalov I, St John A, He Y-W. The antiapoptotic protein Mcl-1 is essential for the survival of neutrophils but not macrophages. Blood 2007; 109: 1620-1626.

12 Ghigo A, Damilano F, Braccini L, et al. PI3K inhibition in inflammation: toward tailored therapies for specific diseases. Bio Essays 2010; 32: 185-196.

13 Yancey PH. Organic osmolytes as compatible, metabolic and counteracting cytoprotectants in high osmolarity and other stresses. J Exp Biol 2005; 208: 2819-2830.

14 Arakawa T, Timasheff SN. The stabilization of proteins by osmolytes. Biophys J 1985; 47: 411-414.

15 Lentzen G, Schwarz T. Extremolytes: natural compounds from extremophiles for versatile applications. Appl Microbiol Biotechnol 2006; 72: 623-634.

16 Galinski EA, Pfeiffer H-P, Trüper HG. 1,4,5,6-Tetrahydro2methyl-4-pyrimidinecarboxylic acid: a novel cyclic amino acid from halophilic bacteria of the genus Ectothiorhodosira. Eur J Biochem 1985; 149: 135-139.

17 Sydlik U, Gallitz I, Albrecht C, et al. The compatible solute ectoine protects against nanoparticle-induced neutrophilic lung inflammation. Am J Respir Crit Care Med 2009; 180: 29-35.

18 Unfried K, Sydlik U, Bierhals K, et al. Carbon nanoparticleinduced lung epithelial cell proliferation is mediated by receptordependent Akt activation. Am J Physiol Lung Cell Mol Physiol 2008; 291: 725-733.

19 Paunel-Görgülü A, Zörnig M, Lötgers T, et al. Mcl-1-mediated impairment of the intrinsic apoptosis pathway in circulating neutrophils from critically ill patients can be overcome by Fas. J Immunol 2009; 183: 6189-6206. 
20 Donaldson K, Tran L, Jimenez LA, et al. Combustion-derived nanoparticles: a review of their toxicology following inhalation exposure. Part Fibre Toxicol 2005; 2: 10.

21 Sydlik U, Bierhals $\mathrm{K}$, Soufi $\mathrm{M}$, et al. Ultrafine carbon particles induce apoptosis and proliferation in rat lung epithelial cells via specific signaling pathways both using EGF-R. Am J Physiol Lung Cell Mol Physiol 2006; 291: L725-L733.

22 Schikowski T, Sugiri D, Ranft U, et al. Long-term air pollution exposure and living close to busy roads are associated with COPD in women. Respir Res 2005; 5: 152.

23 Luo HR, Loison F. Constitutive neutrophil apoptosis: mechanisms and regulation. Am J Hematol 2007; 83: 288-295.

24 Quint JK, Wedzicha JA. The neutrophil in chronic obstructive pulmonary disease. J Allergy Clin Immunol 2007; 119: 1065-1071.

25 Weissenberg A, Sydlik U, Peuschel H, et al. Reactive oxygen species as mediators of membrane-dependent signaling induced by ultrafine particles. Free Rad Biol Med 2010; 49: 597-605.
26 Melley DD, Evans TW, Quinlan GJ. Redox regulation of neutrophilic apoptosis and the systemic inflammatory response syndrome. Clin Science 2005; 108: 413-424.

27 Peuschel H, Sydlik U, Haendeler J, et al. c-Src-mediated activation of Erk1/2 is a reaction of epithelial cells to carbon nanoparticle treatment and may be a target for a molecular preventive strategy. Biol Chem 2010; 391: 1327-1332.

28 Grether-Beck S, Timmer A, Felsner I, et al. Ultraviolet A-induced signaling involves a ceramide-mediated autocrine loop leading to ceramide de novo synthesis. I Invest Dermatol 2005; 125: 545-553.

29 Harishchandra RK, Wulff S, Lentzen G, et al. The effect of compatible solute ectoines on the structural organization of lipid monolayer and bilayer membranes. Biophys Chem 2010; 150: 37-46.

30 Kanapathipillai M, Lentzen G, Sierks M, et al. Ectoine and hydroxyectoine inhibit aggregation and neurotoxicity of Alzheimer's $\beta$-amyloid. FEBS Lett 2005; 579: 4775-4780. 